\title{
Seven ambiguities in explaining the human memory system in the Principles of Neural Science book
}

Seyed Amir Hossein Batouli 1,*

${ }^{1}$ Department of Neuroscience and Addiction Studies, School of Advanced Technologies in Medicine, Tehran University of Medical Sciences, Tehran, Iran

* Corresponding author: Dr. Seyed Amir Hossein Batouli; Assistant Professor; Department of Neuroscience and Addiction Studies, School of Advanced Technologies in Medicine, Tehran University of Medical Sciences, Tehran, Iran; Address: No.88, Italia Street, Keshavarz Boulevard, Tehran, Iran. Email: batouli@sina.tums.ac.ir

\begin{abstract}
Memory is probably one of the most complex cognitive functions of the human, and in many years, thousands of studies have helped us to better recognize this brain function. One of the reference textbooks in neuroscience, which has also elaborated on the memory function, is written by Prof. Kandel and his colleagues. In this book, I encountered a number of ambiguities when it was explaining the memory system. Here, I am sharing those points, either to find an answer for them, or to let them be a suggestion for our future works. Prof. Kandel has spent most of his meritorious lifetime on studying the memory system; however, the brain is extremely complex, and as a result, we still have many years to comprehensively understand the neural mechanisms of brain functions.
\end{abstract}

Keywords: Human Memory; Long-term Memory; Episodic; Implicit; Explicit 


\section{About Principles of Neural Science book}

Prof. Eric R. Kandel, as provided his lifetime story in his "In Search of Memory" book[1], is born in Vienna, 1929. He received the Nobel Prize in Physiology or Medicine in 2000 for all his contributions and achievements on studying the mechanism of memory storage in the brain. Around twenty years before his Nobel Prize, in 1981, he and his colleague, Prof. James H. Schwartz, wrote the book Principles of Neural Science. This book is now in its $5^{\text {th }}$ edition and is more than 3 times larger than its first edition[2]. I always considered the Principles of Neural Science a holy book in neuroscience; to my astonishment, I noticed similar expressions about this book elsewhere, such as calling it the "Bible of Neuroscience".

As a researcher who is interested in human memory[3,4], and in particular the long-term memory (LTM), I studied this book (the $5^{\text {th }}$ edition) and in particular the three chapters of it (chapters $65,66,67$ ) which are on memory. During my studies, some parts of these chapters seemed ambiguous to me, i.e., being insufficiently explained or being in contradiction with the other parts of the book. As a result, in here, I am sharing with you those questions of mine about this book, and I hope either to find out that it was my insufficient knowledge which misguided me, or we find some further explanations about these questions in the next edition of this book or in others' future studies.

The focus of this manuscript has merely been on this book. In other words, there might be answers to my questions in other references; however, regarding such a textbook should be stand-alone, I did not refer to other resources. The aim of the current paper is only to open a discussion on the different aspects of the human memory system, and I would be grateful if experts of the field help us younger researchers to enrich our knowledge about the memory system. The quotations from the book are marked with "asterisks", with the page numbers mentioned in [brackets]. There are 10 questions in total, under the seven categories as provided in below.

\section{Site of memory storage}

It is raised in the book that "the brain does not have a single long-term store of explicit memories" [p.1446], but the "memory storage involves many different parts of the brain" [p.1442]. Kandel et al. also added that "the ultimate storage site for all declarative memories is thought to be in the cerebral cortex" [p.1487], "knowledge is stored in a distributed manner in the neocortex" [p.1451], "storage of any item is widely distributed among many brain regions" [p.1446], and "over time, explicit memories are transferred to different regions of the neocortex" [p.1461]. It is also read that "old memories are not stored in medial temporal lobe (MTL), but in various other 
cortical regions" [p.1448], and "MTL plays a temporary role in consolidation of memories, and after a long period the MTL is not needed as memories can be retrieved directly from cortical regions" [p.1448].

Question1: The above statements are concerning the site of long-term memory storage in the brain, and suggest it to be over the cortex. However, I could not observe an evidence in the book for these claims; neither an animal nor a human study. The site of LTM storage is a substantial issue, and I guess it needs additional confirmations.

\section{Using neuroimaging devices}

The proofs for my above question might have been obtained through the neuroimaging works. It is mentioned in the book that "PET and fMRI allow us to scan the healthy brain in the process of building new memories or retrieving existing memories, and thus to identify the specific regions that are active during different processes" [p.1447]. Examples in the book were "fMRI in healthy people showed that activity increased in the right hippocampus when spatial information was recalled, and in the left hippocampus when words, objects, or people were recalled" [p.1445], or "PET and fMRI studies of subjects whom were asked to recall or recognize previously studied words or pictures showed activity in the anterior and lateral prefrontal cortex" [p.1449].

Question2: I believe, when a brain area is observed active during the retrieval of a memory, that area is not necessarily eloquent for the memory, and could only be involved in a cognitive function associated with the memory. For example, recalling a scene would activate/need the visual processing areas of the brain to process that information; however, this activation should not be interpreted as that area being the site of memory "storage". As is mentioned in the book, "storage refers to the neural mechanisms and sites by which memory is retained over time" [p.1447], and it seems neuroimaging studies could only provide evidence about "how different categories of knowledge are represented in the human brain" [p.1451], and not the site of memory storage.

Here is an example. If by opening a water tap we can fill a glass, and if opening a tap is always significantly associated with the glass being filled, could we deduce that the tap is the place of water "storage"? Similarly and in assessing the human memory system, if running a memory task is statistically associated with the activation of a particular brain area, could we infer that the active brain area is the site of memory "storage", or it should be concluded that it is only part of a chain of brain functions for a successful memory?

\section{Hippocampus role in episodic memory}

Rodent studies which assessed spatial memory and the role of hippocampus in these tasks, are reported in the book. These studies considered the spatial memory tasks as a kind of episodic memory. It is read in the book that "Hippocampus is important for spatial representation; in mice and rats lesions of the hippocampus interfere with 
memory for space and context, and single neurons in the hippocampus encode specific spatial information" [p.1445]. It is also stated that in the water maze test, "if the mouse locate the platform and remember its position based on the spatial contextual cues- markings on the walls of the room that the pool is located-, this task would require hippocampus" [p.1503], whereas "in the non-contextual version that the platform is raised above the water level or marked with a visible flag, permitting the mouse to directly navigate to the platform using brain pathways, the task does not require hippocampus" [p.1503]. Furthermore, it is mentioned that the "hippocampal pyramidal neurons fire in place fields even if the LTP is blocked, which shows the sensitivity of these cells to the spatial location" [p.1510].

Question3: Episodic memory is a vital human function; however, the book seems to have a very narrow look into that: only rodent studies and only spatial tasks are considered. It is mentioned in the book that "long-term memory of a stably formed place field requires the animal to attend to its environment, as is the case for explicit memory in humans" [p.1511]. It seems we need much further information particularly about other aspects of the episodic memory in humans. In addition, based on the above statements in the book, the hippocampus seems to be the central brain structure in episodic memory; however, this seems to be in contrast with the other parts of the book, as provided in below:

The book mentions several examples of the patient H.M., whose hippocampus was resected in a surgery. Despite his lack of hippocampus, and the hippocampus being mentioned in the above rat studies to be important for retrieving episodic memory, he had a flawless ability in retrieving his older episodic memory: "he had LTM for events occurred before the operation" [p.1443], "his semantic memory was preserved" [p.1445], and "he could recall childhood memories, as well as many experiences of his lifetime" [p.1448].

It is also added that the main distortion in H.M. was not his working memory: "H.M had normal WM, for example he could repeat a telephone number for seconds to minutes because of his intact WM" [p.1445], "indicating that the MTL is not necessary for transient memory" [p.1443], but his problem was in the ability to form new memories, or the encoding ability. "He could not recognize people after surgery, even he met them several times" [p.1445], and "he had difficulty in forming and retaining new conscious (episodic and semantic) memories, of their personal experience or the meaning of new concepts" [p.1446]. "The activation of MTL in encoding of stimuli that were later remembered is observed compared to those that were forgotten" [p.1448], suggesting the role of MTL in encoding process. 
Question4: Is hippocampus necessary for having access to older episodic memory? H.M. did not have this brain structure but could remember his older memories, whereas the animal studies suggest that an impaired hippocampus deteriorates retrieval of episodic memory. Kandel mentions that "the observations in H.M. and other amnesic patients with damage to the MTL suggest that old memories are not stored in MTL" [p.1448]. This contradicts with the results of animal studies which report the spatial information (as a kind of episodic memory) to be stored in the hippocampus: "the hippocampus stores declarative information in a more stable form for periods ranging from days to weeks to years, up to a lifetime" [p.1487], and "PKM $\zeta$ is required for the maintenance of LTP but is not involved in its initial induction" [p.1503].

Question5: It is mentioned that "the hippocampus stores declarative information in a more stable form for periods ranging from days to weeks to years, up to a lifetime" [p.1487]. It seems H.M. is a counterexample for this claim.

Question6: The book pointed out that "the retrieval of contextual or event details associated with episodic memory showed activity in the MTL, particularly in the hippocampus" [p.1449]. How could this be interpreted when the hippocampus was not necessary for the retrieval of episodic memory in patient H.M.? Is it only because "MTL activity is thought to facilitate the activation of neocortical representations that were present during encoding" [p.1449]?

\section{Explicit memory in animal}

As observed in the book, episodic memory was mostly studied in rodents, and by assessing the spatial memory. One feature of an explicit memory is its being declarative, and as it cannot not be assessed in animals, the book points out that episodic memory is identified in animals by the being-conscious feature of it. As "consciousness cannot be studied empirically in the mouse" [p.1511] either, "selective attention, which is required for conscious recall, is examined instead" [p.1511].

Question7: Regarding the "being declarable" feature of an episodic memory, should we name this memory differently when being studied in animals?

\section{Size of memory storage}

It is stated in the book that "one of the features of LTM is that it seems to have an almost unlimited capacity; there is no known limit to the amount of information in LTM storage" [p.1447]. One evidence provided for that claim was "a memory system that automatically retained every detail of every experience, such as what happened in Shereshevski, who was filled with highly detailed memories of his past experiences" [p.1458]. 
However, based on the mechanisms reported for the storage of explicit memory in the book, "if synaptic connections could only be enhanced and never attenuated, synaptic transmission might rapidly saturate, the strength of the synaptic connection might reach a point beyond which further enhancement is not possible" [p.1513]. As "individuals are able to learn and store new memories throughout a lifetime" [p.1513], the book suggests that the solution for this paradox is "a mechanisms to downregulate synaptic function to counteract LTP" [p.1513], which is called long term depression. "The LTD suggests an anti-hebbian learning rule: synapses that do not contribute to the firing of a cell are weakened" [p.1513].

Question8: Should we consider an unlimited LTM capacity in humans? If yes, probably the current mechanisms which explain the storage of LTM need further modifications, as apparently, and based on the book, the current mechanisms need LTD for a continuous memory storage, and this equals erasure of our older memories, which does not seem to necessarily happen.

\section{Differences between implicit and explicit memories}

"Implicit memory, also known as procedural or non-declarative memory, takes the form of an unconscious memory, and is an automatic manner" [p.1446, 1461]; it includes "knowledge that are acquired without conscious effort, and guides behaviour unconsciously" [p.1452]. "Implicit memory could only be retrieved during performance and not consciously" [p.1485]. On the other hand, "explicit memory, also known as declarative, is conscious" [p.1446, 1461], and "is the conscious recall of information" [p.1487].

As is mentioned in the book, it seems the episodic memory depends on the MTL structures, whereas the implicit memory does not. Evidence include a "greater activity in the MTL during encoding of stimuli that were subsequently remembered compared to those that were forgotten" [p.1448], and the "episodic learning being dependent on the interaction between cognitive control processes in the PFC and associative binding mechanisms in the MTL" [p.1448]. The disabled encoding ability in patient H.M. is another evidence, and similarly the musician, Clive Wearing, whose hippocampus and temporal cortex were affected, "did not have memory for events or people he met a few minutes earlier" [p.1461], which showed "impairments in his episodic memory encoding", whereas he "could still play on the piano, showing his intact procedural memory" [p.1461].

On the other hand, "priming, as a type of implicit memory, does not depend on the MTL" [p.1452]. "The implicit memory is independent of the hippocampus, such as patients with a damage to the MTL whom could learn to read mirror-reversed text" [p.1453]. Other evidence were the "neural circuits that initiate habit, motor skills and conditioned learning are independent of the medial temporal lobe system, which is responsible for explicit 
memory" [p.1453]; "learning of sensorimotor depending on basal ganglia, cerebellum, and neocortex; dysfunction of the basal ganglia impairing learning of motor skills; and lesions in the cerebellum impairing motor learning" [p.1453]; "skills to depend on structural changes in motor neocortex, such as expansion of the cortex relevant to fingers in musicians" [p.1453]; and "long term storage of implicit memory requiring neocortex for priming, striatum for skills and habits, amygdala for learned fear, cerebellum for learned motor skills, and reflex pathways for non-associative learning” [1461].

Question9: Based on the above statements, the implicit and explicit memories have at least two differences: being dependent on the MTL structures, and being conscious. Those memories which are processed in the MTL become conscious, and the memories which do not pass through the MTL are unconscious. Could it be concluded that the MTL structures are central organs for consciousness?

\section{Differences between semantic and episodic memories}

"Episodic memory is the memory of personal experiences or autobiographical memory, and semantic memory is the memory for facts" [p.1446]. Also, "semantic knowledge is distinguished from episodic knowledge in that it is typically not associated with the context in which the information was acquired" [p.1451].

Question10: Would it be reasonable to infer that the semantic memory is a special kind of episodic memory that is independent of the time and space constraints? An episode, in which the content of the information is more vital than the temporal and spatial features of the event of the data collection becomes a semantic knowledge.

\section{Conclusion}

Once again I insist here that I would never be in the position to criticize so many scientific endeavours and achievements of a great scientist such as Professor Kandel and his colleagues. However, I am searching for the answers of my above questions, to achieve a more comprehensive understanding of the human memory system, and any assistance from experts of the field would be appreciated.

Conflict of Interests: There has been a single author in this manuscript, and there is no conflict of interests.

Acknowledgment: I appreciate all the assistance by Mrs. Minoo Sisakhti in the preparation of this manuscript.

\section{References}

[1] Kandel ER. In Search of Memory: The Emergence of a New Science of Mind. 1st ed. New York, USA: W.W. Norton \& Company; 2006.

[2] Kandel ER, Schwartz JH, Jessell TM, Siegelbaum SA, Hudspeth AJ. Principles of Neural Science. 5th 
ed. New York, USA: McGraw-Hill Education; 2012.

[3] Batouli SAH, Alemi R, Khoshkhouy Delshad H, Oghabian MA. The influence of mental fatigue on the face and word encoding activations. Clin Neurol Neurosurg 2020;189:105626.

doi:https://doi.org/10.1016/j.clineuro.2019.105626.

[4] Batouli SAH, Sisakhti M. Investigating A Hypothesis on The Mechanism of Long-Term Memory Storage. NeuroQuantology; Vol 17, No 32019. 